\title{
Pharmacoeconomics as a Management and Financial Strategy in Healthcare and Simulation as a Decision Making Tool for it: The Case of Ozone Therapy
}

\author{
Marco Remondino \\ Department of Economics (DIEC) \\ University of Genova \\ Email: marco.remondino@economia.unige.it \\ Marianno Franzini \\ University of Pavia and President of International Section, SIOOT \\ Email: info@ossigenoozono.it
}

\begin{abstract}
Contributions: both authors scientifically contributed to this article, both substantively and formally. In particular, the paragraph entitled "The Use of Ozone as a Medication and in Different Sectors" can be jointly attributed to both authors, while all the rest of the work is to be attributed to Marco Remondino.
\end{abstract}

\begin{abstract}
Pharmacoeconomics can play a very important role in decision-making for healthcare structures. In fact, it consists of economic evaluation of drugs and treatments for specific diseases. Several techniques exist for this, mainly based on traditional management and financial methods as, for example: cost-minimization analysis, cost-benefit analysis, cost-effectiveness analysis or cost-utility analysis. The present paper proposes the use of agent based process simulation as an additional tool to assess the pharmacoeconomic analysis, in which to incorporate traditional financial tools. This technique allows to compare different treatments ceteris paribus, that is by simulating the effects of different drugs and treatments on the same patient, by comparing both the medical effects and the economic and financial effects. Last, the paper proposes the case of ozone therapy as a powerful use case for this simulative approach, since ozone, a natural gas with antibacterial and anti-inflammatory properties, can successfully be used to treat many diseases as an alternative to other drugs and treatments.
\end{abstract}

Keywords - healthcare, decision making, financial analysis, managerial analysis, pharmacoeconomics, ozone therapy, agent based process simulation

\section{INTRODUCTION}

Decision-making must necessarily be based on prioritization criteria that allow to allocate resources efficiently. Consequently, the health professional must have the tools to set these criteria when choosing between different alternatives.

One of the many determinants that are intimidating the healthcare system is the significant impact that the incorporation of new technologies in the health field has. This is so much so that it is considered one of the main factors responsible for the growth in health expenditure and average health care provision. The increase in expenditure resulting from the incorporation of technological advances has not contributed to the same extent to the production of social value for the populations that support it, nor has there been such a clear benefit for patients [1].

Special mention should be made of the influence that the introduction of new drugs into health organisations has had on this technological development. This premises make it necessary for all those who are part of the health system to collaborate. In several countries, measures are being evaluated by the public administrations to provide greater control over the situation. Among the proposed actions, and in the area of system efficiency and expenditure control, the importance of incorporating medicines into the health system's portfolio of services under cost-effectiveness criteria is highlighted. In the same way, the construction of pharmacotherapeutic guidelines, as a support for decision making, should be based on criteria of evidence and costeffectiveness.

The inclusion of efficiency considerations in decision making seems to merge into an axiom that, although not characterized by its uniqueness, is becoming more and more evident [2]. Since the early 1990s, in Australia and Canada, and especially with the establishment in the United Kingdom of the British National Institute for Health and Clinical Excellence in 1999, known as NICE, which includes evaluation in the selection of health innovations, the adoption of economic evaluation in decision-making has become an imminent necessity, to which an appropriate response of a practical and applicable nature must be given [3]. 
In this sense, pharmacoeconomy [4] seems to emerge, becoming a cardinal discipline in the health scenario. Pharmacoeconomy provides professionals with information on all the economic aspects in the health sector, providing them with a broader perspective in the analysis of all the implications of the drug through specific methodological tools.

Within the pharmacoeconomical analyses, are included economic evaluations, techniques that allow professionals to calculate the efficiency in terms of the relationship between the costs and consequences of alternative interventions [5].

The reality of the application of the pharmacoeconomy in daily practice can be seen reflected in recent studies such as that carried out by Catalá-López [6].

The number of economic evaluations of health interventions is increasing, with a maximum growth in the last five to ten years. The scope of most of the studies analysed was in specialized care. With these data, it seems clear that the use of economic evaluations is becoming increasingly essential in the healthcare sector. The main reasons, documented by the health professionals themselves, that make it difficult to implement effective decisionmaking criteria are, on the one hand, the difficulty of understanding this type of study or the approach that they usually take and, on the other hand, the fact that it is far removed from daily practice. Other arguments that question the credibility of economic evaluations are that these studies are, in most cases, financed by the pharmaceutical industries and arise from the mistaken idea of understanding economic evaluations as purely savings and not quality improvement tools. Once the problems are circumscribed, only the implementation of solutions is possible. Thus, and after defining a framework in which the application of pharmacoeconomics seems to be an increasing value in the healthcare environment, but which still needs to be encouraged, it is necessary to establish instruments that allow to confront the problems that still seem to be felt for their use in clinical decision-making. For this reason, simulation is proposed as a tool both to teach pharmacoeconomics and a to actually compare the effectiveness and actual economic impact of different alternatives.

In particular, the use of ozone in medical therapies is chosen as proposed a use case for the present analysis. This is because ozone, a natural substance, has proven to be an outstanding alternative to standard treatments in a wide spectrum of diseases, with effective results and potentially with direct and indirect cost reductions both for the medial structure and for the patients.

The first part of the work will introduce pharmacoeconomics as a technique for comparing treatments and hence as a management strategy for economic evaluations in the healthcare sector. The second part will deal with ozone and its use in medicine. The discussion will propose several simulation techniques that could support pharmacoeconomic analysis, with specific regard to the use of ozone in alternative to other standard treatments.

\section{PHARMACOECONOMICS AND ECONOMIC EVALUATIONS WITHIN HEALTHCARE}

The application of the knowledge and techniques of business management and economic evaluation to the field of healthcare makes it possible to focus the analysis on the treatment of the problems posed by an appropriate prescription both from the point of view of effectiveness in terms of health and of efficiency, thus obtaining a social benefit at a reasonable cost. Pharmacoeconomics is therefore a useful tool for measuring the effects of decisions on available resources and also on the possibilities for quantifying the health impact of a health intervention. As a result of the current approach of the health authorities to pharmaceuticals, the pharmaceutical industry worldwide has begun to take an interest in these analytical techniques and to use them to develop its research-development, production and marketing strategies for its products. The economic evaluation of medicines is not a panacea for the problems faced by health professionals on a daily basis, but it does make it possible to reduce the arbitrariness of decisionmaking on the use of available resources, so that it is an indispensable instrument for improving health practice and achieving better results at an affordable cost for a country's economy [7].

A medicine [8] can be defined as a pharmaceutical preparation or formulation used for the prevention, diagnosis and/or treatment of a disease or pathological condition, or to modify physiological systems for the benefit of the person to whom it is administered. Although medicines alone are not sufficient to provide adequate care, they play an important role in protecting, maintaining and restoring health.

Medicines are not only an essential component of health care, a symbol of treatment, but also a commodity and a commodity of modern industry, a commodity and a commodity to be traded and sold.

As an input for health actions, they represent a cost that has significant repercussions on official budgets and on people's budgets.

From the economic point of view, medicines are consumer goods that are those products made to be used, both for productive consumption and for personal consumption. Therefore, the drug can be defined as a product, as any material good that has value for the consumer or user, and is capable of satisfying a claim and reporting a monetary income to the producer.

Medicines are important inputs in health services and are the subject of commercial transactions. They are obtained from community pharmacies and a high proportion of their income is used to purchase them. As the relationship between the medicinal product as a marketing object and its 
value in use is so decisive that it gave rise to the concept of the medicinal product as a "social good" [9].

The modalities and reasons for medical consumption are conditioned by a number of interacting factors, including: therapeutic and prescribing practices, the attitude of patients towards physicians, the perception of the population with regard to medical properties, the economic resources of individuals, the availability of public and private funds for their purchase and distribution, and the promotional activities of the pharmaceutical industry. Employment is also determined by cultural aspects and other specific characteristics of the consumer population, such as prevalent diseases and available technological and economic resources.

On the other hand, in our days, the attention of the health is the subject of intense commercial activity which leads to the excessive consumption of its services and, in particular, to the use of all technological resources. These conditions reinforce the role of medicines.

The lucrative nature of the industrial activity of drug production and marketing has led to major diversions such as the production of unnecessary, ineffective or toxic drugs, irrational prescription, self-medication, etc. This, in turn, affects teaching, information and consumption activities.

The medicinal product has become a complex element whose use involves considering not only the pharmacotherapeutic, health and social aspects but also those of an economic, industrial, financial and commercial nature. Thus, there is a need to devise a policy that harmoniously links industry and commerce on the one hand, and the consumer, the doctor and the health authority on the other. Most countries [10] face a shortage of resources in the area of health, which forces them to contain, redirect and rationalize their expenditures.

This situation and the absence of a defined policy and programmes lead to serious problems in financing the health care system, with the consequent increase in private spending and a decrease in resources allocated to pharmaceutical assistance. This is even more serious in developing countries, where there is also a shortage of hospitals and health centres that serve the neediest sectors of the population. Insufficient availability of medicines, medical and surgical supplies and other inputs in public services has a negative impact on the quality of care and the use of services.

This situation has resulted in the current emphasis on assessing the social and economic impact of medicinal products, while taking into account the technical and scientific aspects of their efficacy, safety and quality. In this context, new proposals have been generated for the development of the pharmaceutical area under the concept of socioeconomic evaluation of medicines $[1,3]$.

Among the various aspects that should be considered in this context before authorizing their introduction to the market, the following may be mentioned: potential for inducing self-medication, therapeutic indications in relation to prescription habits, possibilities generated by the forms of presentation of the physician in terms of compliance or noncompliance with the treatments and the influence that the system of care will have on their use, as well as the cost of the treatment in relation to others. Evaluations of this nature would be important in health care systems for the use of smaller forms.

With regard to the economic and financial aspects of the use of medicines, the evaluation should be based on a relative consideration of cost, benefit and risk. The incorporation of new drugs into a country's health system and market should take into account these financial and economic aspects of drug use.

In order to evaluate the use of medicinal products, from a more global point of view and not only in pharmacotherapeutic terms, it is necessary to carry out economic evaluations of medicinal products. Economic analyses of this type, referring to specific drugs or drug classes or types, should be published as any other research result and be publicly accessible or, perhaps, it would be necessary for such information to be provided in conjunction with information on efficacy and safety in relation to indications, dosage of use, treatment time and so on. In fact, it is a question of the comparison between pharmaceutical products and other treatment methods with regard to their therapeutic properties and the costs of their use as alternative native methods, and the feasibility of applying economic concepts of cost/benefit to decisionmaking in the pharmaceutical sector [1].

These types of pharmacoeconomic studies have introduced relative drug evaluation mechanisms, whereby different medicines are compared and therapeutic substitution between therapeutically similar products (even if they are made up of different active ingredients or different pharmaceutical forms and have the same active ingredients) is assessed.

The economic evaluation of drugs - whose synonym is pharmacoeconomics - can be expressed as the application of economic analysis to the field of medicine; and it can also refer more specifically to the application of economic theory to pharmacotherapy. Since all evaluation is based on comparison, economic evaluation of drugs could be defined as the determination of the efficiency (cost-benefit ratio) of a drug treatment and its comparison with other options in order to select the most cost-effective treatment.

In this way, the economic evaluation of measures can contribute to harmonizing the growing health needs of the population with the available economic resources. The selection of a given therapeutic option according to its efficiency; that is to say, considering its costs and benefits simultaneously, was a considerable advance compared to other more partial criteria.

The efficiency criterion [11] is applicable by different actors in different areas of healthcare domain. Recent international experiences $[12,13,14,15]$ show that economic evaluation techniques for medicines are becoming 
increasingly important in health decision-making, providing invaluable help in making them based on more rational, objective and transparent criteria.

The economic evaluation of medicines usually employs the traditional methods of economic analysis [16], which means that all professionals working in the field and interacting information from it need basic knowledge of the terms and concepts that are currently the common denominator of both economic evaluation and health economics. For this reason, pharmacoeconomics is an activity that requires multidisciplinary teams and, in fact, a high proportion of analysts who carry out studies in this field are not economists but come from the field of health sciences, statistics, operational research, etc., and have often not had a formal apprenticeship in economic disciplines.

In this way, the knowledge of the pharmacoeconomic concepts on the part of professionals working in both the health system and the pharmaceutical industry makes it possible to introduce an economic culture into their usual tasks; that is, to consider the existing relationship between the costs and the results of the pharmacotherapy used (Figure 1).

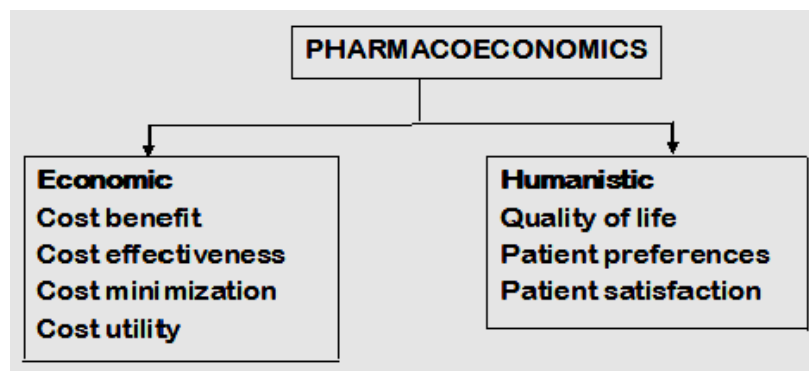

Figure 1. The two sides of Pharmacoeconomics

From a clinical perspective, the use of a technology is justified if its effectiveness (the achievement of a target under real conditions) or at least its effectiveness (the achievement of a target under ideal conditions) is positive. However, from an economic perspective, the emphasis is on efficiency.

Thinking in terms of efficiency implies considering the effectiveness of a process in relation to the resources it requires. The reason is obvious, given that resources are limited with respect to their possible applications, the fewer resources are needed to achieve a given objective, the better; as this will make more resources available for other health purposes.

At present, and given the high cost of developing a new product, there is a clear vocation and orientation within pharmaceutical companies to provide increasingly efficient, effective, better tolerated and higher quality drugs, which will represent a clear advance and improvement within the available pharmaceutical arsenal. For this reason, within the pharmaceutical industry, medical economic evaluation analyses are increasingly being designed and carried out throughout the entire life cycle, both during the early stages of their development and once they have been commercialized. This fact reflects the need to prioritize the investment of existing resources, and to choose drugs that are the most cost-effective and therefore achieve the best therapeutic benefit at the lowest possible associated cost.

In industrialized societies capable of regulating and controlling the production costs of health goods and where health coverage is almost total, pharmacoeconomics can certainly help to improve clinical decision-making [1], especially when it comes to choosing between several similar treatments. Every exercise, however, must be focused on the perspective not only of controlling costs, but also of improving efficiency in order to achieve equity in access to health services.

\section{THE USE OF OZONE AS A MEDICATION AND IN DIFFERENT SECTORS}

In 1896, Tesla [17] issued a patent for an ozone generator using metal plates. He founded the Ozone Tesla Company and began manufacturing these units. His clients were naturopaths and allopates who welcomed this invention well. The respiration of ozone through olive oil and other oils was widely practiced at the time. Tesla produced a gel made from ozone bubbles through olive oil until it solidifies, and sold it to doctors.

Let's think about what happens when our body lacks oxygen and is assaulted by fatigue, circulatory disorders, digestive difficulties, muscle pain and the collapse of the immune system. From here you can see how beneficial oxygen ozone therapy is which is a medical practice deputed to carry oxygen and especially ozone (three oxygen atoms) to increase the ability of our body to use this gas.

The fields of use are vast: from the now famous application in the hernia of the disc to the most recent one in the field of oncology and pain therapy. And again in dementias; in the treatment of bacterial and viral inflammations and in the aesthetic and dental field.

Ozone oxygen therapy finds its development and validity in being a therapy with low costs and with very high benefits. It has been practiced for years, but it has always remained a niche therapy. It is an "intervention" that has no side effects, as it is performed with oxygen, which is one of the fundamental elements for the life of our organism that is converted into ozone, gases with high healing properties. Furthermore, ozone therapy has no contraindications even if it must be practised by specialists who know the treatment protocols, the dosages to be used, the number of sessions and the frequency with which they can be repeated.

In order to precisely define the toxicity of a gas, it is necessary to establish:

1. Concentration

2. exposure time

3. route of administration 
Ozone therapy is one of the methods with the lowest risk of side effects (Jacobs clinical trial 1981). Fundamental rules for operating in complete safety are:

1. correct administration: scrupulously following the protocols

2. use of suitable equipment certified and revised

3. the use of a gas with a low risk of side effects. Not to be inhaled

4. Not too pain. Good ozone therapists must not cause pain. Pain can trigger even uncontrollable vagal reactions

The routes of administration of ozone are as follows:

- Great Autohemoinfusion or, better, Systemic Oxygenation: metabolic rebalancing, better use of body oxygen, correction of circulatory ischaemia, detoxification from toxins and free radicals, increased resistance to stress, immunomodulating action, treatment of bacterial/viral infections, even antibiotic-resistant, action on the microcirculation, including the brain, neurodegenerative diseases.

- Small Autoemoinfusion: immunomodulating, antiviral, herpes zoster, and simplex, acne, fibromyalgia, antidepressant action.

- Subcutaneous: muscle decontracting, cellulite, reactivation of the venous circulation, lipolytic action.

- Intramuscular: decontracting, herniation and disc protrusion. - In particular: limbs, arthrosis. - Insufflation (rectal, vaginal, uterine, urethral): Gae replacement, analgesic treatment, treatment of infections, fibromyalgia.

- Topical route: ulcers, sores, muscular contractures, acne, venous insufficiency, oral treatments, cellulite, treatment of skin spots.
- Hydropinic: body water rebalancing, dysbiosis, helicobacter pylori.

Ozone is a natural gas universally known for its microbial properties. It kills bacteria by cell lysis by attacking the protective membranes with an oxidative mechanism and altering their internal enzymes, without leaving chemical residues. Viruses are first inactivated and then physically destroyed, while in others Ozone enters into the depths and damages viral nucleic acids by preventing the replication mechanism. Moreover, as far as organic molecules are concerned, they are attacked by ozone with the oxidizing action of atomic oxygen $(\mathrm{O})$ or through the formation of unstable compounds (Ozonoids) that lead to the breakage of the molecules themselves.

Ozone is also able to reactivate circulation and microcirculation. In particular:

- it increases erythrocyte deformability and filterability, because it breaks down the long chains of fatty acids through controlled lipoperoxidation;

- it increases the production of 2.3diphosphoglycerate with greater oxygen transfer to peripheral tissues;

- it reduces platelet aggregation and plasma viscosity;

- it reactivates energy metabolism at mitochondrial level with an increase in the production of ATP.

The primary purpose is to try to interrupt the involutive cycle of degenerative events by inducing the cells to reactivate the synthesis of Oxidative shock proteins (osp). A good percentage of patients show a significant improvement (figure 2) due to a beneficial influence on circulation, oxygenation, mood tone and attention.

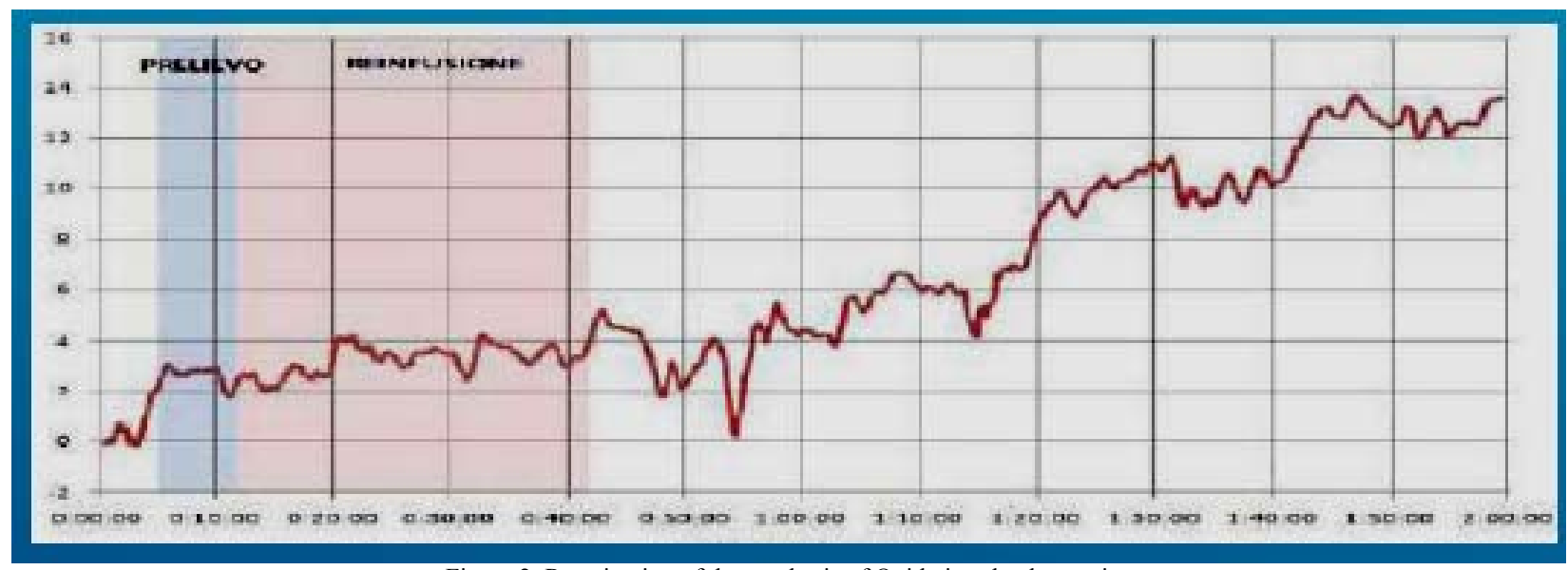

Figure 2. Reactivation of the synthesis of Oxidative shock proteins

The interpretation of the test concerns the following points:

- Following treatment with ozone, an increase can be observed significant concentration of oxygenated hemoglobin
- The effect is only visible at least one hour after the start of the treatment.

At the same time, non-oxygenated hemoglobin remains almost constant, indicating an increase in cerebral oxygenation for the same oxygen consumption. 
Therefore, a situation improved in the functionalities because it is better to capture the oxygen present.

The whole thing translates into:

1. improvement of brain microcirculation

2. increased attention

3. improvement of cognitive activities and memory

4. reduction of neuromuscular spasticity

5. anti-aging

Ozone also has an anti-inflammatory and pain-relieving action; improving oxygenation, inhibiting leukocyte and platelet activation and adhesion, inhibits the activation of phospholipase A2, cyclooxygenases and metalloproteinases. This will inhibit the bio-humoral inflammatory response. In particular we have: synthesis;

Inhibition of prostaglandin pro-inflammatory

- Inhibition of the release of bradychidine and algogenic compounds;

- Neutralization of endogenous ROS and stimulation of local production of antioxidant enzymes.

Another medical field in which ozone therapy has great success is hernia disc treatment [18]. As regards oxygenozone injections into the spinal column area, the ministerial circular of 20 January 2005 reiterates that '[...] intradiscal and/or intraforaminal injections can only be carried out experimentally (and therefore free of charge) in accredited hospital facilities and always in the context of controlled clinical trials $[\ldots]$ '. The only route of administration approved by the Higher Institute of Health is the paravertebral route. In figure 3 , the results are shown from a study conducted on 7.253 patients.

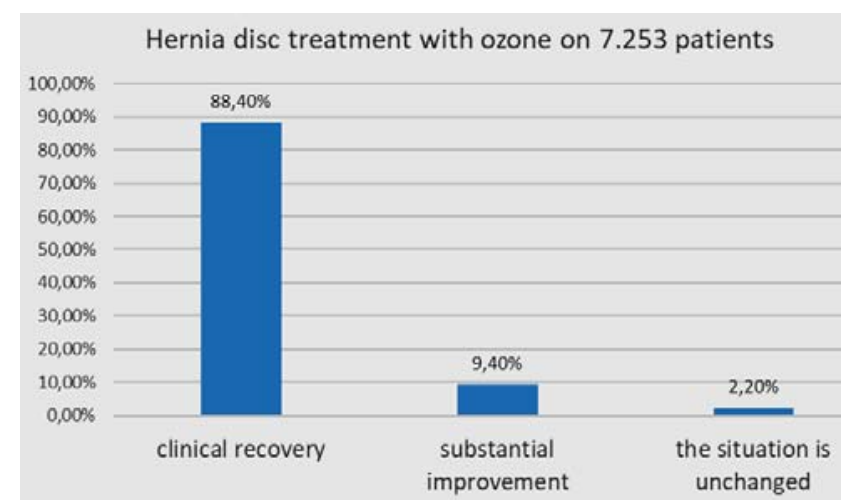

Figure 3. Results from a study about hearnia disck treatment with ozone

A very topical issue where ozone can also be very interesting is that of antibiotic resistance [19]. The phenomenon of antibiotic resistance in bacteria attacking our organism is becoming increasingly worrying. This is demonstrated by the recent scientific reports [20] from the United States on the presence, in the urine of a 49-year-old from Pennsylvania, of a strain of escherichia coli resistant to Colistin, the most powerful antibiotic in circulation, the drug used in extreme cases of infection of bacteria capable of "killing 50\% of those affected", according to experts of the American society for microbiology.

According to Thomas R. Frieden [21], director of the Centres for Disease Control and Prevention, a public health organization in the United States, "we have entered a postantibiotic era in which we may have to rely less and less on these drugs that, for decades, have saved millions of lives.

The main culprit of antibiotic resistance is the mcr-1 gene, first identified by Chinese researchers in November 2015 [22], which can put bacteria in a position to resist the most potent chemical drugs. The study had identified the gene in common but potentially fatal bacteria such as escherichia coli and klebsiella pneumoniae, responsible for pneumonia and blood diseases. A gene that could unfortunately spread easily in other bacterial strains, creating new types of so-called superacteria.

Faced with what could take on the form of a global emergency in the coming years, there are two possible solutions: to continue investing in the production of increasingly effective antibiotics, or to encourage the use of alternative therapies capable of complementing chemical drugs. One of these alternatives is ozone therapy, since there is no bacterium, virus or fungus that can withstand a correct dose of ozone, administered in the right times. In fact, at the cellular level, bacterial resistance to antibiotics depends on several factors, first of all the structural change of the superficial envelopes of the bacterial cell, which reduces the penetration of antibiotics. In gram-negative bacteria resistance may be due to alterations in the protein membrane through which many antibiotics penetrate.

In other cases, the bacterium is able to expel the drug, which has already entered the cell, thanks to efflux pumps that operate coupled to a particular type of protein. It can also happen that the target of the antibiotic is altered and therefore no longer recognized by the drug or that the antibiotic loses its biological activity because inactivated by some specific enzymes produced by both bacteria of gram positive and negative type.

About $5.7 \%$ of all types of klebsiella pneumoniae isolated in Europe in 2014 were resistant to all groups of antimicrobial agents normally used. In addition, more than $80 \%$ of these were reported in Greece and Italy.

A problem which, again, ozone therapy can remedy. Ozone, in fact, kills bacteria by cell lysis, attacking their protective membranes, with an oxidative mechanism, and altering their internal enzymes without leaving chemical residues. A mechanism similar to that used by white blood cells during bacterial phagocytosis.

Ozone is a powerful oxidizer and an important disinfectant that acts on microorganisms through the oxidation of their biological material. It has been shown that ozone can be used as an antibacterial agent in various forms such as ozonized saline solution, ozonized water, ozone 
associated with particular oils or other types of substances, and, more frequently, the gaseous mixture of oxygen and ozone. Most studies on oxygen-ozone therapy have shown that ozone, when used in vitro, could destroy all types of bacteria, positive gram and negative gram. In addition, this research indicates that the susceptibility of microorganisms to antibiotics increases when ozone is used, as does the effectiveness of the immune system.

\section{AGENT BASED SIMULATION AS A POWERFUL SUPPORT TOOL FOR PHARMACOECONOMICS}

The healthcare context is particularly complex as it is characterized by high quality standards, low management costs and management of assets, material and immaterial, bound to the criteria of efficiency, effectiveness and economy defined in the central planning following the various reforms of the sector; the rules of "supply" and "demand" at the base of this sector cannot simply be traced back to the classic functional paradigms of commercial systems but are determined in a complex manner by a series of factors difficult to analyse, summarize and define. In this context, in order to be able to manage these systems in the best possible way, new techniques of analysis and management are needed, and this is the focus of the study. The most widespread methods of analysis and management of systems in the healthcare environment are ex-post or exante; these techniques are not effective in a dynamic and complex context such as healthcare, as they do not allow to have real-time information on the behaviour of the system analysed or to bring the necessary corrective actions reasonably quickly. Real time data analysis techniques exist, but are poorly applied in this context, given their difficulty of use in environments with high variability and uncertainty. In the healthcare context, when we speak of a management and control system, we commonly speak of Business Intelligence systems; with this term we refer to systems that allow the collection of data with the purpose of analysing the past activities of an organization in order to understand its functioning, its problems and try to evaluate its performance and estimate its future functioning. One of the main criticalities that is created with these systems is that they are fed with data collected asynchronously to the time of the evaluation and therefore function as internal reporting systems that can provide information on the past behaviour of the system and predict its future behaviour on the basis of information that does not necessarily reflect the current behaviour of the system and therefore, may not make sense from the point of view of management analysis.

The purpose of the present paper is to propose the use of agent based process simulation (ABPS) as a decision making tool for pharmacoeconomic analysis and, particularly, for the analysis of ozone therapy treatments when compared to traditional ones. The purpose is twofold, since the simulation can be a great decision making tool, but also a great descriptive tool to practically show the outcomes of a certain strategy.

First and foremost, Agent-based simulation (ABS) is selected as the base method for simulating situations of pharmacoeconomics. This is because ABS has proven to be a tremendously useful technique for modelling complex systems [23]. Through agent-based simulation, the modeller explicitly recognizes that complex systems, and in particular social systems, are the product of individual behaviours and their interactions. What distinguishes agent-based simulation from other modelling techniques is the way in which the first abstraction of the real system is constructed and, consequently, the formal model, especially using a metaphors based approach [24] (figure 4), a powerful method able to represent a real situation in a simulation models by means of metaphors and then interpreting the simulated results by means of an inverse function of those metaphors. In formal models constructed through agentbased simulation, the basic components of the actual system are explicitly and individually represented in the model.

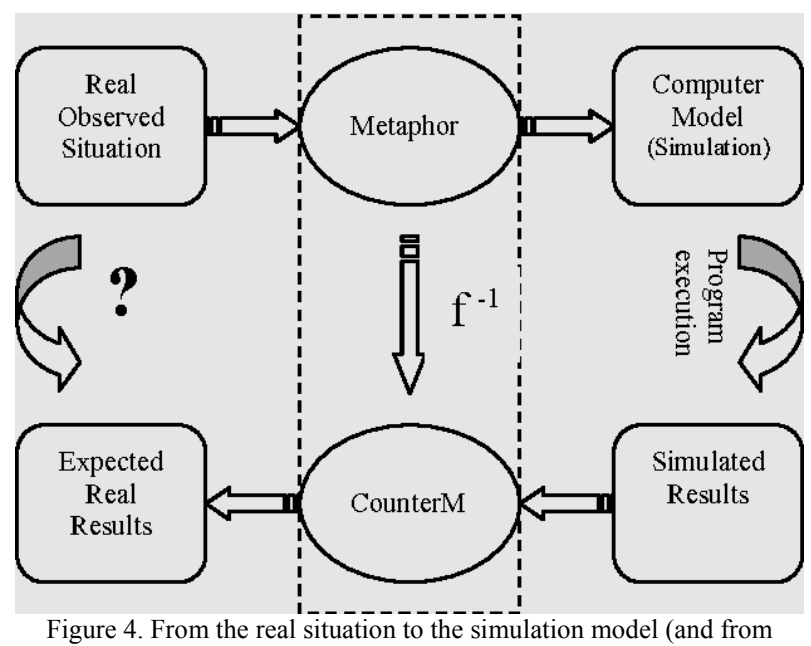

simulated results to realistic ones)

The use of ABS is complemented by some parts of the model (the most deterministic ones) that can be modelled by a process based approach. This is what is referred to as ABPS approach [25].

The use of simulation as a tool for pharmacoeconomic analysis allows the inclusion of a greater number of variables and entities, as well as the representation of their behaviour over time, thus allowing the model developed to be more closely linked to reality than any other modelling technique such as mathematical ones or even medical ones. One of the main components in a simulation model are the entities that are going to be simulated, for the case of the pharmacoeconomic analysis, most of the times the entities are the patients that suffer from the disease under study, however, it may be the case that the entities of the model are of another type, for example medical units or doctors that attend to a certain condition, this depends on the approach 
that one wishes to give to the study in progress. Unlike a Markov model, where the focus is primarily on the overall model results or states, in a multi agent based model the patient could be an explicit element of the study. Each of the entities within a simulation model has different attributes that determine the way in which they behave during the simulation.

These attributes have certain initial values and are modified over time depending on the rules established when designing the model, for example, when studying a person with diabetes, one of the attributes that they have is the weight they had at the time they were diagnosed with the disease, however, this variable changes over time depending on the type of treatment the patient follows, the complications that occur or the eating habits they have. It is important to stress that the rules of change and implications for patient attributes must be studied and clearly defined so that the results obtained are valid and can be interpreted appropriately. It is important to emphasize that each of these changes can be assigned a monetary value that serves as an indicator in the pharmacoeconomic analysis.

When using agent based simulation as an analysis tool, the rules of change of state of patients should be included, depending on some global variable, for example, in the particular case of ozone therapy applied to osp, the synthesis of Oxidative shock proteins may function as the main indicator of the health of a given patient, depending on this level will be the treatment received, the complications that may arise due to the condition of this disease, the type of treatment to be followed or even the mortality rate. As mentioned above, all of these changes need to be recorded in order to determine what the costs of treating a given disease are. A pharmacoeconomic analysis can also be used to study the allocation of non-monetary resources. For example, how many beds are needed for an epidemic that may occur at any given time, or how many specialist doctors must be on hand to care for patients with some type of cancer. Through the use of agent based simulation it is also possible to make a study of different scenarios, for example, when dealing with some type of disc hernia, it is possible to design a model in several versions, where the change is the type of treatment a patient receives, and the results also impact other factors, like for instance the time they'll need to stay away from work. Hence, the simulation is presented as a tool for comparison between different scenarios.

Use a agent based model for pharmacoeconomic analysis of complex diseases has a number of advantages over other methods. As already mentioned, it is a less restrictive and more flexible technique than decision trees and the Markov strings with discreet status space, as it allows to enter a number of variables as well as the rules that produce a change in the system over time, thus generating a model that is more closely linked to the reality. Besides, it is possible to use probability distributions in different ways, depending on the of the input data available (and historical data). In addition to the fact that through the realization of several replications (coeteris paribus) a scenario which is statistically significant can be obtained. It is a model that is easy to implement as there is no need to force the evolution of the disease in mutually exclusive states or force it to change at certain time intervals. In addition to the fact that it can reduce the time needed to draw conclusions instead of conducting medical studies that may take more time and require a greater allocation of resources. It is a straightforward model, since all the components and entities are part of it. the model, contrary to what would happen in the other methods in which patient characteristics are not a definite part of the model, plus that accurate time control is being maintained. It is possible to vary the structure of the model and thus to analyse several scenarios, which are which would not be possible with clinical trials or other methodologies. Global and differentiated statistical estimators of costs are obtained based on in the resources consumed, the simulated scenario and the processing followed. Statistical techniques can be used to analyse the outputs of the simulation experiments. Time is explicitly considered within the event simulation the behaviour of variables and entities can be modelled in a discreet manner, thus making it possible to the length of the simulation. Simulation also allows the analysis of different scenarios and the implications of the same, thus being a powerful tool for decision making. By simulating what happens in a health care system by treating a certain a more realistic view of the implications of this disease is acquired. complications or doses of medicines, such as the complications involved. necessary for their treatment, influencing the actions to be taken such as the prevention or faster response to certain events. Although there is no complete information available on the functioning of the by means of the simulation it is possible to make reliable estimates about the performance.

\section{QUALITATIVE DESIGN AND RESULTS OF A SIMULATION MODEL FOR OZONE THERAPY PHARMACOECONOMIC ANALYSIS}

As stated in previous sections, ozone therapy has a very wide range of powerful and effective applications, hence it's impossible to think about a single (generalist) simulation model to embrace them all. For each specific case, a simulation model has to be created and tuned, as to variables, parameters, rules and entities. The purpose of the present section is to qualitative describe the simulation framework that could be used for a pharmacoeconomic evaluation of ozone as an alternative treatment of several, different diseases.

Patients are randomly generated, each of whom is assigned physical characteristics based on the information obtained from past clinical studies. Each patient is replicated to simulate it in each of the two designed scenarios (treatment with ozone vs treatment with traditional therapy). 
A third scenario could be used, which is the one in which the patient does not follow any type of treatment for the disease, so to benchmark both results against a "zero treatment", which could serve as a basis for comparison of both costs and complications for both the other treatments.

For each of the scenarios, rules are defined that model the appearance of complications depending on the time the patient has suffered from the specific disease and whether or not she has undergone any treatment before. A statistically significant number is defined for the total number of patients to be simulated. As well as the number of replicas that will be made of the model, this in order to obtain statistically significant estimators.

A compilation of costs of the different treatments, presented throughout the simulation, is made by statistical analysis and a comparison is carried on between the scenarios.

The whole simulation logic is represented in figure 5 .

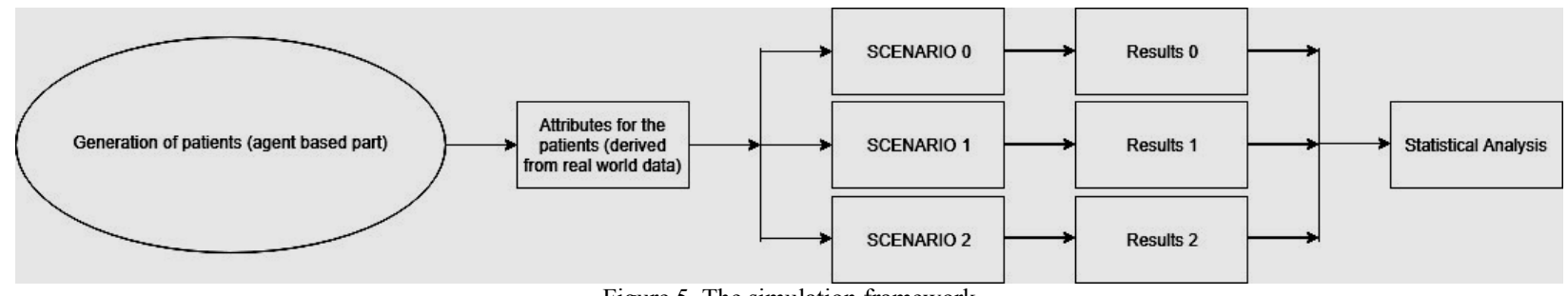

Figure 5. The simulation framework

\section{CONCLUSION AND FUTURE DIRECTIONS}

The purpose of the present work is twofold. First, it aims to discuss the use of simulation as a tool for pharmacoeconomics, a powerful decision making analysis for healthcare sector. To this extent, pharmacoeconomics is presented in detail, with its features and fields of applications. Besides am agent based approach is proposed for the simulation, enriched by parts managed with a more traditional process simulation approach and, schematically, a logical flow is proposed, starting with the generation of patients, the definition of specific attributes for them, derived from real world data and the consequent passing through three simulated scenarios. "Scenario 0" is the base one, that is the one where the patient is not undergoing any treatment for her disease, while "scenarios 1 and 2" are those where the patient is treated with the two alternative drugs/medical cares.

The second purpose of the work is to propose to the scientific community the pharmacoeconomic evaluation, by means of simulation, of ozone as a treatment in alternative to other, traditional, medical cares. For this reason, the paper synthetically presents a range of uses for ozone, a natural gas, where it proved to be an effective alternative to other treatments, being safe and effective as an antibacterial, antiinflammatory, decontracting and oxygen vehicle [26].

Since there are more and more medical applications for ozone therapy, it can be really interesting to evaluate its use by means of pharmacoeconomics and this makes it the ideal candidate for the novel approach proposed in this paper.

Future works will take care of real situation, by applying the proposed methodology to use cases, hence assessing both the medical side of the issue (the validity of ozone as a cure for several diseases) and the management side, that is the possibility of using a cheaper medication. Not to mention that ozone, being a natural gas, has no impact on the environment, making it a potential source of sustainability.

Hence simulation applied to pharmacoeconomics appears to be a really interesting decision making method for healthcare structures and the case of ozone therapy needs to be analysed through this methodology.

\section{REFERENCES}

[1] Gray, J. A., and Larry W. Chambers. "Evidence-based healthcare: how to make health policy \& management decisions." Canadian Medical Association. Journal 157.11 (1997): 1598.

[2] Lancsar, Emily, and Jordan Louviere. "Conducting discrete choice experiments to inform healthcare decision making." Pharmacoeconomics 26.8 (2008): 661-677.

[3] Sculpher, Mark J., et al. "Whither trial - based economic evaluation for health care decision making?" Health economics 15.7 (2006): 677-687.

[4] Areda, Camila Alves, Roni Cléber Bonizio, and Osvaldo de Freitas. "Pharmacoeconomy: an indispensable tool for the rationalization of health costs." Brazilian Journal of Pharmaceutical Sciences 47.2 (2011): 231-240.

[5] Drummond, M. F., M. J. Sculpher, and G. W. Torrance. "O Brien BJ, Stoddart GL." Methods for the economic evaluation of health care programmes 3 (2005).

[6] Catalá-López, Ferrán, et al. "Burden of disease and economic evaluation of healthcare interventions: are we investigating what really matters?." BMC health services research 11.1 (2011): 75.

[7] Welte, Robert, et al. "A decision chart for assessing and improving the transferability of economic evaluation results between countries." Pharmacoeconomics 22.13 (2004): 857-876.

[8] Dumit, Joseph. Drugs for life: how pharmaceutical companies define our health. Duke University Press, 2012.

[9] Ruger, Jennifer Prah. "Health and social justice." The Lancet 364.9439 (2004): 1075-1080.

[10] Shinjo, Daisuke, and Toshiharu Aramaki. "Geographic distribution of healthcare resources, healthcare service provision, and patient flow in Japan: a cross sectional study." Social science \& medicine 75.11 (2012): 1954-1963.

[11] Atkinson, Roger. "Project management: cost, time and quality, two best guesses and a phenomenon, its time to accept other success criteria." International journal of project management 17.6 (1999): 337-342. 
[12] Stahl, James E. "Modelling methods for pharmacoeconomics and health technology assessment." Pharmacoeconomics 26.2 (2008): 131-148.

[13] Schuette, Wolfgang, et al. "Treatment decisions, clinical outcomes, and pharmacoeconomics in the treatment of patients with EGFR mutated stage III/IV NSCLC in Germany: an observational study." BMC cancer 18.1 (2018): 135.

[14] Porter, Martina L., and Alexa B. Kimball. "Pharmacoeconomics of Systemic and Biologic Therapy in Dermatology." Biologic and Systemic Agents in Dermatology. Springer, Cham, 2018. 83-91.

[15] Miot, Jacqui, and Michael Thiede. "Adapting Pharmacoeconomics to Shape Efficient Health Systems en Route to UHC-Lessons from Two Continents." Frontiers in pharmacology 8 (2017): 715.

[16] Samuelson, Paul Anthony. "Foundations of economic analysis." (1948).

[17] Elvis, A. M., and J. S. Ekta. "Ozone therapy: A clinical review." Journal of natural science, biology, and medicine 2.1 (2011): 66.

[18] Vanni, Daniele, et al. "Intraforaminal ozone therapy and particular side effects: preliminary results and early warning." Acta neurochirurgica 158.3 (2016): 491-496.

[19] Neu, Harold C. "The crisis in antibiotic resistance." Science 257.5073 (1992): 1064-1073.
[20] Fernandes, Miriam R., et al. "First report of the globally disseminated IncX4 plasmid carrying the mcr-1 gene in a colistin-resistant Escherichia coli ST101 isolated from a human infection in Brazil." Antimicrobial agents and chemotherapy (2016): AAC-01325.

[21] Frieden, Thomas R. "The future of public health." New England Journal of Medicine 373.18 (2015): 1748-1754.

[22] Liu, Yi-Yun, et al. "Emergence of plasmid-mediated colistin resistance mechanism MCR-1 in animals and human beings in China: a microbiological and molecular biological study." The Lancet infectious diseases 16.2 (2016): 161-168.

[23] Siebers, Peer-Olaf, et al. "Discrete-event simulation is dead, long live agent-based simulation!." Journal of Simulation 4.3 (2010): 204-210.

[24] Remondino, Marco. "Agent Based Process Simulation and Metaphors Based Approach for Enterprise and Social Modeling." ABS 4 Proceedings (2003): 93-97.

[25] Remondino, Marco. "Agent based process simulation for management and economics." Economic Complexity Online Journal (2003).

[26] Franzini, Marianno, Luigi Valdenassi, and Giulia Ionita. "First evaluations of oxygen-ozone therapy in antibiotic-resistant infections." Ozone Therapy 1.1 (2016): 5-7. 